NOTAS 


\title{
NOTAS TEÓRICAS EN TORNO AL CONCEPTO DE POSTPRODUCTIVISMO AGRARIO
}

\author{
Xosé A. Armesto López \\ Departamento de Geografía Física y Análisis Geográfico Regional \\ Universidad de Barcelona
}

\begin{abstract}
RESUMEN
El objetivo de este artículo es analizar el surgimiento del término postproductivismo y aportar una base teórica que pueda servir para el posterior empleo de este concepto por parte de los investigadores rurales interesados en estudiar este fenómeno en España. Así, después de una primera parte introductoria, se explican cuestiones acerca del origen y ubicación científica del término. Además se inscribe en el contexto rural a partir del cual deriva la materia agraria, de la misma manera que desde la Unión Europea (UE) se pretende que se entiendan los nuevos campos europeos desde las premisas de la sostenibilidad y la multifuncionalidad. De este modo, el postproductivismo expresa esa nueva ruralidad que se predica nominalmente desde las instituciones públicas, a pesar de que la realidad sobre el terreno sea bien diferente ya que los sistemas productivistas siguen gozando de una gran aceptación entre la inmensa mayoría de los agricultores y ganaderos de toda Europa.
\end{abstract}

Palabras Clave: Postproductivismo, productivismo, agricultura, desarrollo rural, sostenibilidad, multifuncionalidad.

\begin{abstract}
This paper aims to analyze the emergence of the term postproductivism and to contribute a theoretic base that could serve to the later use of this concept by the Spanish rural scientists interested in this subject. In this way, after an introductory first part the text includes questions related with the origin and the position of the postproductivism in the social science movements. Furthermore it is analyzed the postproductivist's agrarian viewpoint starting for the rural context, in the same way the European Union (EU) ende-

Este artículo forma parte de la Tesis doctoral del autor Agricultura ecológica y postproductivismo: un estudio comparado entre Cataluña y Galicia. Dirigida por la Dra. Roser Majoral y el Dr. Rubén Lois.

El presente artículo ha sido realizado en el marco del proyecto de investigación sobre Desequilibrios territoriales, mercados de trabajo y áreas marginales de Cataluña financiado por la CICYT (proyecto ref. BSO20013095) y con un Ajut de Suport a la Recerca dels Grups Consolidats del II Pla de Recerca de la Generalitat de Catalunya (Grup de recerca d'Anàlisi Territorial i Desenvolupament Regional, 2001SGR-00016) ambos dirigidos
\end{abstract} por la Dra. Roser Majoral.

Fecha de recepción: 23 de marzo de 2004. Fecha de aceptación: 15 de octubre de 2004 . 
avour to show the European fields under the scope of sustainability and multifunctionality. So, the postproductivism express this new rurality that is enunciated by the public institutions, despite of that, the reality is sufficiently different because of the productivist systems continue having a great acceptation between the European farmers.

Key words: Postproductivism, productivism, agriculture, rural development, sustainability, multifunctionality.

\section{Introducción}

En este artículo se abordan cuestiones relativas al carácter conceptual del postproductivismo. Éste forma parte de un trabajo más amplio en el que se analizaron las relaciones que se establecen entre las agriculturas ecológicas de dos territorios plenamente diferenciados desde la perspectiva agraria como son Galicia y Cataluña y el propio carácter del postproductivismo. Así, este trabajo no debe ser contemplado desde la perspectiva de la Geografía rural clásica, en la que los compartimentos estancos eran la base de las argumentaciones. Se pretende que el hecho de formular conexiones transversales con otras disciplinas como la economía, la sociología o la propia filosofía, que trascienden el mero hecho geográfico, puedan servir como fundamento para futuros trabajos de investigación relacionados con estos nuevos paradigmas productivos y territoriales.

De modo general se puede decir que la denominada «tercera revolución agrícola» ${ }^{1}$ (Bowler, 1992) es la basada en la industrialización de la agricultura, o lo que es lo mismo la fase productivista que tiene su máximo exponente en los años que van desde el final de la II Guerra Mundial hasta los finales de los años ochenta del siglo pasado y que se sustenta sobre una concepción tecno-económica. Es en ese momento cuando empieza a notarse la coexistencia de dos paradigmas referentes al hecho productivo agrario: el ya mencionado productivista, y el que autores como Bowler (1996) denominan postproductivista. Las características de la fase productivista, época en la que la agricultura se convierte en el gran enemigo de la biodiversidad y en la que dos palabras para definirla pueden ser explotar y subsidiar (Lockwood, 1999), pueden resumirse en:

a) la creación de economías de escala a nivel de explotación, con el consiguiente aumento de la dimensión de las explotaciones;

b) una creciente dependencia de los insumos provenientes de otros sectores de la economía, puesto que se genera la necesidad de compra de fertilizantes, pesticidas, piensos y maquinaria, se trata pues, de un incremento de los flujos energéticos y también de los desechos contaminantes. Hay autores como el propio Lockwood (1999) que consideran que ésta es la diferencia clave;

c) la substitución de recursos, ya que se pasa de basarse en la tierra y el trabajo, por fundamentarse en el capital, con el consiguiente aumento de las tasas de paro y el descenso de la población rural;

1 Bowler identifica dos revoluciones anteriores: la primera se remonta 10.000 años atrás y coincide con la domesticación de buena parte de las especies animales y vegetales. La segunda de ellas habría que buscarla entre el S/XI y el XIV en Europa Occidental época en la que se mejoran aperos como el arado, comienzan a sustituirse los bueyes por caballos como animales de tiro y se crea un mercado agrocomercial en consonancia con el desarrollo urbano. Fundamentalmente es en los últimos setenta u ochenta años cuando la mecanización, los agroquímicos y la agroindustria hacen su aparición masiva en la agricultura occidental, pudiendo hablar por tanto de Tercera Revolución. 
d) la puesta en práctica de una organización dirigida y controlada por las grandes empresas de sectores, en inicio, ajenos a la agricultura ${ }^{2}$;

e) la modificación de componentes naturales y culturales, como, por ejemplo, la eliminación o modificación de los sistemas de separación tradicional entre parcelas y explotaciones, los cambios en los usos del suelo en terrenos con humedales naturales, o la pérdida de patrimonio y tradición en las formas de edificación;

f) el papel subsidiario de la biodiversidad por el que se hacen aparecer nuevos cultivos y variedades, ejerciéndose un control bioquímico de los organismos y dejando extinguirse razas tradicionales puesto que se consideran desaprovechables, ineficientes o simplemente pasadas de moda;

g) el importante peso específico de las ayudas institucionales públicas para garantizar precios a consumidores y productores;

h) el incremento de la diferenciación entre los papeles del hombre y la mujer en el mundo agrario ${ }^{3}$.

Estos puntos definitorios, que se relacionan entre sí, se han ido extendiendo durante el siglo XX de manera implacable a lo largo del planeta pero con una implantación desigual tanto geográfica como sectorial, quedando segmentadas de este proceso áreas económicamente marginales, y por el contrario ejemplificando esta gran industrialización sectores como el hortícola o el ganadero intensivo. Además a todas estas cuestiones de importancia se les ha sumado últimamente una preocupación más de carácter global, la agricultura productivista y su implicación en el cambio climático, algo que ya está comenzando a ser referido y estudiado por algunos investigadores (Alexandratos, 1995; Holloway e Ilbery, 1998; Olmos, 2002).

A esa notable industrialización de la agricultura y su problemática intrínseca hace referencia Bowler (1992) cuando define magistralmente las consecuencias secundarias de cada una de sus dimensiones estructurales; así derivadas de la intensificación de la producción aparecen el crecimiento de la deuda agraria, el incremento de la intensidad energética y por tanto de la dependencia de los combustibles fósiles, la destrucción del medio ambiente y los ecosistemas, el desarrollo de cooperativas de abastecimiento y, la aparición de la sobreproducción en los mercados domésticos. En lo concerniente a la concentración de las unidades productivas las consecuencias son la aparición de nuevas relaciones sociales en las comunidades rurales, el desarrollo de cooperativas de mercado, la propiedad corporativa de la tierra, el incremento en la diferenciación de las explotaciones según su tamaño y las consecuentes diferencias por lo que respecta a sus ingresos y posibilidad de ayudas públicas y, el aumento de las desigualdades entre las explotaciones de diferente tipo y localización así como de su tratamiento desde el punto de vista de las políticas estatales y regionales. Finalmente, y por lo que respecta a la especialización de la producción, se destaca que los alimentos son consumidos fuera de sus regiones de producción, se produce un cambio en la composición de la fuerza de trabajo, aparece una mayor rigidez estructural en la producción, se eliminan las actividades menos lucrativas y se incrementa el riesgo de fallos en el sistema.

2 Halweil (2002) ilustra esta situación al hablar de los granjeros norteamericanos en 1950, los cuales de cada dólar que se gastaban sus compatriotas en comida recibían la mitad, mientras que la situación para 1997 reflejaba que de ese mismo dólar solo recibían siete céntimos, quedándose los noventa y tres restantes en manos de los diversos intermedediarios (procesadores de alimentos, comerciantes, proveedores de fertilizantes y plaguicidas, etc.). De hecho, esta situación es aplicable en la actualidad a cualquier país del mundo.

3 Trauger (2001) considera que las mujeres agricultoras, al menos en el caso de Minnesota, tuvieron más dificultades para participar en la agricultura productivista y por eso son ellas las que lideran la transición postproductivista. 
Respecto a estas tres dimensiones de la agricultura J. Gómez Mendoza (2001: 113-114) resalta que están dirigidas al sector agroalimentario y que han sido favorecidas desde una PAC totalmente sectorial que ha promovido históricamente unos precios de garantía elevados. Para esta autora, mediante estas tres dimensiones, la agricultura productivista «ha roto con el modelo natural y territorial, al asumir la dependencia de inputs externos, pasar del policultivo con ganadería al casi monocultivo en muchas regiones, y condenar a muchas tierras a la marginalidad económica y demográfica. (...)».

Ese puede ser, por tanto, un buen punto de partida para empezar a definir y analizar un concepto tan controvertido en la actualidad en lo que a su extensión y significación se refiere como es la agricultura postproductivista. De partida no parece poder afirmarse que nos encontremos a escala europea en una nueva fase productiva, pero sí es evidente que algunos vectores empiezan a modificar su dirección lo que ha llevado a algunos autores (Marsden, 1992; Bowler, 1996; Evans, 1996) a hablar de una «Transición postproductivista». De hecho, estas nuevas tendencias, que como marco geográfico se sitúan en lo rural, están profundamente ligadas a lo urbano y diseñan una nueva ruralidad que en un futuro, no demasiado lejano, puede hacerse más plausible. Ese cambio en el mundo rural hace que las definiciones que hasta el momento se han efectuado de estos territorios sean cada vez más cuestionables, de ahí que el marco epistemológico para estudiarlos necesite de una renovada aportación que pueda ayudar a todos los investigadores (geógrafos, sociólogos, economistas, etc.) a interpretar y estudiar esa nueva realidad.

En algunos enclaves de determinadas regiones europeas el funcionamiento del mundo rural en la actualidad es muy diferente del que había hace solo una veintena de años. En palabras de Bonnamour (2001: 36-37) «El cambio de la sociedad nos parece tan profundo que el mundo rural no volverá a ser lo que fue, algo que los ancianos todavía lamentan». El proceso de cambio incide en aspectos que hay que tratar de comprender en su conjunto, desde una perspectiva geográfica integradora, puesto que abarcan relaciones territoriales, económicas, sociales, demográficas, económicas, culturales y ambientales ${ }^{4}$.

Estos cambios relacionales hacen que el mundo rural comience a ser visto no como una simple superficie de producción sino como un espacio que puede aportar y aporta una serie de valores de sumo interés para una sociedad global cada vez más urbanizada. Estos «nuevos» valores tienen mucho que ver con el paisaje, la ecología ${ }^{5}$, la cultura, la justicia social y la alimentación saludable, conectando de forma directa con un concepto que en la última década está en boca de todos, el desarrollo sostenible. Relacionado con esta concepción aunque con diferencias significativas en el planteamiento genérico aparece el concepto de desarrollo rural, en él es donde hay que inscribir el concepto del postproductivismo.

Respecto a ese desarrollo rural Sanz (2001) indica que el marco social en el que se inscriben las nuevas tendencias de desarrollo viene caracterizado por: a) nuevos modelos de organización industrial donde destacan la flexibilidad organizativa y la capacidad de innovación y programación; b) reestructuración espacial de las actividades económicas y renovación de los criterios de localización de éstas; c) incremento y dominio del tiempo de ocio; d) centralidad del conocimiento como fuente de desarrollo y de formulaciones políticas sobre la sociedad. Todos esos temas comienzan a situar cual es la verdadera dimensión a

4 No sólo hay que contemplar la interacción disciplinar si no que como señalan Ramos y Romero (1994) hay que comprender que el problema agrario es solo una de las vertientes de la crisis que sufre el mundo a finales del segundo milenio.

5 Todas las leyes y procesos que ocurren en los sistemas ecológicos o naturales influyen de un modo directo en las actividades económicas, sobre todo en la agricultura (entendida bajo su acepción más amplia), en la que el binomio biología - economía aparece, si cabe, todavía más fuerte. 
considerar cuando se habla de postproductivismo. De cualquier manera hay que tener en cuenta lo que comenta Halweil (2002), cuando explica para el Worldwatch Institute, que en todos los países del mundo se ha usado durante tanto tiempo la producción como único factor definidor del éxito, que incluso ahora se hace difícil para los diversos actores implicados en el uso del medio rural llegar a comprender el verdadero alcance de la problemática generada por el productivismo.

\section{Las conexiones e inspiraciones de lo «post»}

Para definir la palabra postproductivismo, en primer lugar, hay que tener en cuenta que se trata de un préstamo del inglés. Efectivamente, el origen de la aplicación al espacio rural de este término está en las Islas Británicas, y hasta hace muy poco tiempo, en el año $1996^{6}$, su empleo por parte de los científicos sociales españoles era prácticamente nulo. Esta es, por tanto, una razón fundamental para comprender que el marco teórico es de procedencia anglosajona. Autores como Bowler, Ilbery, Halfacree, Evans, Lowe o Ward desde comienzos de la década de los noventa del siglo pasado emplean esta palabra para definir las nuevas pautas del mundo rural inglés desde la Geografía y la Sociología.

El término desde el punto de vista léxico aparece compuesto por la partícula de origen latino post, cuyo significado es «después de o detrás de», como algún autor señala «(...) dicho prefijo presenta un uso social tan generalizado, por preceder a casi cualquier cosa que ha perdido una parte de su contenido. A la postre, es un concepto tan ambiguo como en su momento lo fueron los de postmodernismo, postfordismo, sociedad postindustrial, ..., con los que existen además, ciertas concomitancias.» (Rubio, 1999: 39). En la misma línea Peterson (2000) enuncia que esta nueva fase que está comenzando está enmarcada en lo postindustrial, lo postfordista o una fase desorganizada del capitalismo donde los estados nacionales se debilitan.

La profusión de prefijos como «pre-», «pro-», «neo»- o «post-» pueden también dar un carácter imperfecto o inconcluso al sustantivo que acompañan; «la mayoría de las veces resultan ser términos provisionales, muletas conceptuales destinadas a ayudar a los hombres a moverse en su incipiente circunstancia (...)» (Rodríguez, 1997: 1). Por su parte, para Kumar y Bauman el postproductivismo se trataría de una reacción al productivismo, empleándose este término más para analizar, estudiar y reincorporar nuevos elementos de juicio en el examen del productivismo que no para definir un concepto novedoso (ob. cit. Rubio, 1999). Hay autores (Rubio, 1999) que consideran de modo general que la orientación productivista es la propia del productor, mientras que el postproductivista es un enfoque deseado por el consumidor. Por el contrario existen autores que clarifican que aunque hay diferencias entre los diferentes agentes a la hora de poner el énfasis, en una parte de los productores también existe una preocupación responsable por la calidad (Ilbery y Kneafsey, 2000).

Este nuevo concepto es el que sirve para definir la situación provocada por la acumulación irracional de excedentes en los almacenes comunitarios y que ha llevado a que se empiece a modificar el reparto de los recursos destinados a agricultura reduciendo los precios de garantía y aumentando los destinados al estudio y conservación de la calidad ambiental en todas sus vertientes. Para Evans (2001: 46) es en los primeros años de la década de los noventa cuando se acuña el término postproductivismo «para transmitir la idea de un cambio importante en las prioridades agrícolas, distinto de la focalización en el aumen-

6 Es en ese año cuando se celebra en Leicester (Reino Unido) el Primer Simpósium de Geógrafos Rurales Británicos y Españoles que apareció un tiempo más tarde publicado con el título «La investigación hispano-británica reciente en Geografía Rural: del campo tradicional a la transición postproductivista». 
to de la producción alimentaria. Desde ese momento el término se ha convertido en una muletilla de moda para resumir un complejo cambio agrario, o incluso rural, desde una perspectiva más general». Este mismo autor periodiza en tres etapas la creación del concepto actual: $1^{\text {a }}$ ) Se identificaba con las estrategias de ajuste de las explotaciones familiares para mejorar su situación. $2^{\mathrm{a}}$ ) Se produce un giro en la percepción de factores como la calidad, las prácticas agrarias con respecto al medio ambiente, la regulación y la subvención de la agricultura y la multifuncionalidad. $3^{\mathrm{a}}$ ) La etapa actual es en la que se está teorizando sobre el sentido del concepto.

Retomando la teoría del postproductivismo, no se puede dejar de hacer mención a las fuentes epistemológicas donde beben los teóricos de esta nueva fase; como otros movimientos de diversa índole «post» el origen se encuentra en el postmodernismo, esa corriente filosófica que M. A. Rodríguez (1997) califica de un modo atrevido como «mezcla extraña de conservadurismo, pragmatismo, individualismo burgués y pesimismo; todo esto convenientemente impregnado de informática, conforme a las pautas culturales impuestas por la tercera fase de la revolución industrial», y que surge en buena medida por factores negativos propios de la segunda mitad del siglo XX como la división del mundo en dos bloques y la consiguiente amenaza nuclear, el fallo de los acuerdos de Bretton Woods ${ }^{7}$, a principios de los setenta, la aparición de nuevas enfermedades con carácter de peste, las crisis energéticas, las crecientes desigualdades sociales y económicas entre los países del Norte y los del Sur, la conflictividad social generada por la substitución del trabajo por capital, la adopción inadecuada de nuevas tecnologías y, la cada vez más precaria, situación medioambiental. Estas fueron algunas de las circunstancias que hicieron aparecer, desde principios de los cincuenta, experiencias intelectuales que más tarde encajarían en un movimiento que se calificó como Postmodernismo que cristalizó a finales de los setenta y que de un modo u otro pretendía enfrentarse con la incertidumbre que dominaba a las sociedades occidentales.

Según Habermas, en las dos últimas décadas del siglo XX, el cambio de la sociedad occidental radicó en el abandono de la racionalidad iniciada en el siglo XVIII y que definió a la modernidad en tres esferas autónomas: ciencia, moralidad y arte. Desde el año 1996 en que Alan Sokal puso en duda el carácter progresista postmoderno, han sido numerosos los pensadores que han criticado este movimiento y que han acabado por argumentar que el postmodernismo en la actualidad se encuentra en franca regresión y decadencia (Camelo 2000; Rodríguez, 1997).

Desde el punto de vista económico la época moderna se había caracterizado por la dominación del capital sobre el trabajo, por la producción en masa o en serie, el predominio de los mercados masivos y los bienes estandarizados en un espacio jerarquizado ${ }^{8}$. La lógica económica, la ventaja comparativa, el dominio absoluto de la fábrica sobre la sociedad y la dimensión mundial son fundamentales para entender el carácter del fordismo.

La acumulación fordista dependió de formas keynesianas de consumo colectivo. Esta concepción económica que arrancaba a principios del siglo XX en EE.UU entró en crisis en los años setenta, cuando se generó un nuevo modelo de competencia que debía aumentar la diferenciación entre los productos y mejorar su calidad, así es como se produjo una reestructuración que hizo que las pequeñas y medianas empresas ganasen terreno gracias a la descentralización productiva. Por su parte, las grandes empresas, emprendieron un doble camino de desintegración vertical en los aspectos netamente productivos y de aumento de

7 Se hace referencia a la supresión de la convertibilidad del Dólar en Oro y las posteriores devaluaciones de la moneda norteamericana.

8 La estructuración del espacio económico internacional y el espacio jerarquizado Adda (1997) lo relaciona con el equilibrio de las potencias y el concepto de hegemonía. 
competencias en su vertiente más tecnológica. En realidad, las grandes corporaciones han revivido, en los últimos tiempos, cuestiones absolutamente fordistas y tayloristas, como por ejemplo la producción just in time o la bajada de precios de sus productos y la eliminación de la competencia local gracias a sus cambios de escala de producción y al respaldo de las leyes de comercio internacional ${ }^{9}$. El fordismo definido como régimen de acumulación, proceso laboral y norma de consumo fue desarrollado como un concepto de corte familiar capaz de variar expresiones nacionales antes que un conjunto de principios organizativos regidos (Marsden, 1992).

La definición del postfordismo es más compleja, «(...) es todavía un concepto menos coherente, menos estructurado que su predecesor. (...)» (Safón, 1996). Los paradigmas principales de esta nueva visión económica son la especialización flexible, la tecnología de la información, la automatización, la postmodernidad y la globalidad. Autores como Hyman y Streeck (ob. cit. Marsden, 1992) diferencian el término por la producción de calidad diversificada. Se trata por tanto, del comienzo de una nueva época en el desarrollo capitalista. El concepto está íntimamente ligado con el de neofordismo, en el que la variación viene introducida por la diferenciación de los productos, eso sí, con las mismas pautas de regulación que en la era fordista, por tanto este segundo término significa evolución y no ruptura como el postfordismo. Lo que parece evidente es que la actitud del consumidor es lo que ha hecho cambiar el sistema. «(...) la fábrica debe enfrentarse ahora a una sociedad que ya no absorbe todo lo que ésta produce, que no permite la maniobra tradicional de disminuir costes aumentando el volumen de la producción. Una sociedad que resiste al dominio de la racionalidad instrumental propia de la esfera productiva, no consintiendo una programación lineal y obligando la estructura productiva a adecuarse una y otra vez al capricho del mercado (...)» (Revelli, 1996: 3). Este autor va todavía más allá al argumentar que cree que este movimiento postfordista traspondrá los muros de las fábricas y que se convertirá en un movimiento de revolución social que podrá modificar estructuras, instituciones y comportamientos.

Por tanto, el postmodernismo y el postfordismo pueden ser considerados, según sea la visión, como una ruptura con el sistema precedente imperante o bien como una adaptación continuista del gran capital para sobrevivir en el gran negocio industrial. Se puede entender que los dos postulados tienen una parte de certeza, dependiente en buena medida de la escala y el contexto geográfico de aplicación. No hay más que pensar en los pioneros de las producciones alternativas y su trasfondo ideológico y en el lado contrario en las grandes corporaciones industriales y comerciales, máximas exponentes de la llamada globalización, que últimamente han estado aprovechando la «moda verde».

En este último sentido P. Veltz (1999) señala al respecto de la globalización que desde un punto de vista geográfico el proceso de globalización adquiere formas geográficas muy variadas, y que puede apoyarse en una división del trabajo amplia en el seno de una red muy extendida, aunque también puede basarse en concentraciones privilegiadas y en mecanismos de regionalización. Planteando así, la globalización como estrategia de control, no de supresión, de la diversidad, contrariamente a lo que muchos grupos contestatarios exponen cada vez que hay una reunión de algún organismo económico internacional (FMI, G8, etc.). De cualquier modo, no faltan críticas a este proceso desde la Geografía Rural (Hervieu, 1997; Bonnamour, 2001) argumentando que la globalización no es sinónimo de uniformización, si no que por el contrario, acrecienta las diferencias y las desigualdades territoriales, sociales y económicas.

9 Una perspectiva crítica sobre el papel que juegan las grandes empresas y los poderes políticos en la concepción de la sustentabilidad y el desarrollo local puede verse en Chatterton (2002) 
Como consecuencia de todo lo anterior, la expresión postproductivismo rural define una novedad en el estado de estos espacios, estos territorios se están adaptando a una nueva situación ambiental, demográfica, social, económica, territorial y política (Rubio, 1999), temas todos ellos que conforman la Geografía rural. Para explicar como se llega a esta transición, o al menos a la duda sobre la benignidad del modelo productivista Hervieu (1997) señala cuatro razones:

a) la primera es de cariz económico: el incremento de la producción ha conllevado un incremento del volumen de ayuda;

b) la segunda es de carácter ecológico: el despertar de la preocupación por la situación del hábitat de la agricultura;

c) la tercera tiene que ver con la toma de conciencia de la existencia de unos problemas generados por la globalización ${ }^{10}$;

d) la última razón hay que contemplarla desde la escala estatal, en la cual se considera que mantener una política agraria basada en patrones cuantitativos condenaría a muerte a la mayoría de los agricultores.

Esta fase postproductivista de marcado carácter alternativo se caracteriza por la globalización ${ }^{11}$ de la producción y el consumo, la desintegración de la regulación y el soporte estatal, la conciencia de los efectos medioambientales de las prácticas agrarias, el uso creciente de la tierra agraria para otros propósitos y el crecimiento de los consumidores verdes y, por tanto, de la demanda de productos ecológicos y de calidad. Ilbery y Bowler (ob. cit. Trauger, 2001) caracterizan esa transición postproductivista por la producción de productos alimentarios frescos, orgánicos y reconstituyentes destinados a los consumidores verdes en un sistema de producción y distribución descentralizado.

De cualquier modo, esta transición hacia el postproductivismo está adquiriendo en los últimos tiempos tintes que aproximan mucho más estas nuevas modalidades a lo que se podría denominar neoproductivismo; si se analizan las nuevas pautas de distribución y comercialización de los productos ecológicos se puede constatar sin ningún género de dudas que las grandes corporaciones agroalimentarias han reaccionado desde el punto de vista empresarial posicionándose en esos mercados que hasta hace muy poco tiempo habían sido despreciados por su baja implantación. El hecho de que el grupo de consumidores con conciencia medioambiental y preocupación por su salud haya aumentado substancialmente en el último lustro, y que por lo general sean personas dispuestas a pagar más por esos productos, ha servido de incentivo para que las grandes corporaciones se interesen por este mercado en auge. Así, hoy por hoy, se puede decir que el carácter alternativo y postproductivista de estas producciones corre el peligro de que acabe convirtiéndose en una simple adaptación del productivismo.

\section{La valoración de lo rural y la vertiente agraria del postproductivismo}

Al analizar el marco conceptual en el que se sitúa la Transición Postproductivista es de obligada consulta los trabajos que tienen al respecto los británicos Bowler e Ilbery, dos de

10 Esta globalización amenaza con ocultar la historia cultural de los alimentos y cultivos mediante los sistemas de procesado, en muchas ocasiones antinaturales, y los movimientos a grandes distancias a los que se somete a las mercancías.

11 Murdoch y Miele (1999) consideran a este respecto, que los procesos de innovación que promovieron la globalización también hicieron que la estandarización se convirtiese en la norma en la producción y también en el consumo. 
los pioneros en la explicación de este nuevo fenómeno del mundo rural. Para el primero de ellos existen tres razones políticas por las que se puede hablar de transición postproductivista en la agricultura europea: $1^{\text {a }}$ la reforma de la PAC de 1992. De hecho, en ese mismo año aparece el V Plan de Acción Medioambiental de la Comisión Europea donde la agricultura figura como una de las cinco actividades económicas en el que la sostenibilidad es un objetivo político a conseguir ${ }^{12} ; 2^{\text {a }}$ las negociaciones del GATT acabadas en $1993 ; 3^{\text {a }}$ la cada vez mayor convergencia entre la política agraria y la política medioambiental en la UE. Estas mismas tres consideraciones son esgrimidas por otros autores como Ramos y Romero (1994) para definir el paso del productivismo a lo que ellos denominan con una terminología diferente el Ruralismo, y que es fácilmente identificable con el postproductivismo. El impacto que puede generar esta situación en la agricultura europea hay que analizarla desde tres dimensiones que ayudan a conceptualizar el término (Bowler, 1996):

a) El paso de la intensificación a la extensificación; con este planteamiento se logran importantes logros en la reducción de insumos contaminantes, sobre todo cuando se aplican en subsectores como el de los cereales o la ganadería., se consigue un mayor respeto por la naturaleza y unos productos de mayor calidad.

b) La transición de la concentración a la dispersión; la situación idónea se produce cuando las explotaciones más grandes se subdividen, pero esta es la dimensión que menos ha prosperado, puesto que no aparece de una manera demasiado clara en las principales políticas agrarias y de desarrollo rural (Rubio, 1999). Con esta situación se pretenden reducir los impactos que generan la excesiva densidad de explotaciones en áreas concretas mediante el reequilibrio territorial.

c) El camino de la especialización a la diversificación; en esta dimensión los agricultores tratan de buscar nuevas salidas a su trabajo para evitar la polarización, así diversifican sus productos tanto desde planteamientos agrarios como no agrarios. Esta es una estrategia que se ha empleado tradicionalmente en épocas de crisis en las explotaciones agrarias.

Para medir la implantación de estas tres dimensiones hay que analizar los siguientes factores; en el primer caso, el de la extensificación, el factor definitorio es el Margen Bruto Stándard por hectárea de tierra cultivada; en la dispersión hay que analizar los cambios en el tamaño de la explotación, y, finalmente en la diversificación se tienen en cuenta los índices de especialización de los usos del suelo principales (cereales, cultivos industriales, frutas, hortalizas, viña y pastos).

Las previsiones apuntan a que si se llegan a asumir esas tres dimensiones por parte de los agricultores y ganaderos europeos, se producirán importantes cambios tanto a nivel continental como a nivel de explotación, y tanto desde el punto de vista económico como desde el social y el ambiental ${ }^{13}$. Así, la reducción de excedentes de los productos continentales, el mayor interés de los agricultores en la retirada de tierras, la reducción de las cantidades y los costes de los insumos agrarios, la mejora de los parámetros medioambientales del medio rural $^{14}$ y la mejor comprensión por parte de los consumidores del trabajo y la función de los

12 Las otras actividades económicas que deben perseguir este controvertido concepto son la industria manufacturera, la energía, el transporte y el turismo.

13 Últimamente han aparecido voces discordantes como la de Evans (2001) que ponen en duda el avance de la corriente postproductivista, argumentando que el análisis de estas dimensiones, al menos en el Reino Unido, es que la fuerza productivista sigue intacta.

14 Para Trauger (2001) la Transición Postproductivista es un cambio en la conciencia productiva y en la ética de base y puede asociarse a movimientos que buscan alcanzar la sostenibilidad. 
agricultores son cuestiones que están estrechamente ligadas con el desarrollo y la adopción de técnicas postproductivistas. Esta aceptación técnica a escala de explotación depende de varios elementos:

1) Las condiciones físicas, variables como la aptitud agronómica de los suelos o la climatología del lugar, son importantes para esa aceptación.

2) La distancia física y social de los centros de consumo masivos donde la demanda de productos de calidad es más alta, también se revela como algo que hay que considerar en el análisis.

3) La disponibilidad de mano de obra más o menos abundante en los períodos concretos en que más se necesita y la edad, formación y conciencia ambiental de los agricultores propietarios, puede ayudar a una implicación mayor de los agentes productores.

4) El tamaño de la explotación, así la respuesta a los estímulos del postproductivismo no se prevé igual para las grandes explotaciones de corte industrial que para las pequeñas explotaciones de índole familiar.

5) La existencia de organismos públicos y privados de fomento y regulación de este tipo de producciones son los responsables de proveer de información y soporte a los que ya se han decidido por esta alternativa y también a los posibles interesados en un futuro en la adopción de esa filosofía productiva. En esta misma línea y también relacionado con el tercer punto Trauger (2001) cree que la adopción de esta agricultura que ella llama divergente es realizada por aquellos agricultores que quieren continuar cultivando, o que quieren comenzar a cultivar sin entrar en el sistema del complejo agroindustrial.

Reemprendiendo la explicación de la crisis del modelo productivista que dio lugar a estos nuevos episodios de producción y consumo, en concreto, en el medio rural, la situación provocó y continúa provocando nuevas experiencias para los visitantes, trabajadores y residentes, estos fenómenos han contribuido a que aparezca un amplio muestrario de mercancías que poseen un mayor valor añadido, no solo desde el punto de vista económico si no también social y ambiental. Se puede decir, por tanto, que el paisaje rural tiene en la actualidad unas relaciones entre los procesos de producción y consumo más variadas que en la etapa plenamente productivista, Benediktsson (2000) define esta característica con el término «polivalencia rural». Esa polivalencia rural se puede comprobar en la siguiente figura en la que se muestra como en el contexto postproductivista no solo la vertiente agrícola fundamenta la economía, el medio ambiente y la sociedad, sino que sujetos como los productos artesanos y tradicionales, el turismo y la preservación de la floresta acaban de conformar ese nuevo carácter postproductivista. Todos ellos configuran el aspecto de los nuevos campos europeos en los que la función productiva clásica queda solapada por los nuevos usos y requerimientos que se le dan al mundo rural.

Barajando la dimensión agraria y medioambiental es imposible no hacer alusión al concepto de calidad, marca genérica representativa de lo que el postproductivismo pretende. La calidad aparece como eje fundamental de la nueva agricultura europea y pretende sustituir la búsqueda del aumento de la producción. «La noción de calidad es, para la década en que vivimos, lo que la noción de cantidad fue para los años de posguerra» (Hervieu, 1996:117). La relación entre mundo rural, sobre todo por lo que a la agricultura y a la ganadería respecta, y calidad no está exenta de peligros, así Marsden (1992) avisa sobre la agotabilidad de los recursos en el medio rural y de cómo un exceso de consumo y de demanda afectaría ineludiblemente a la calidad de esos productos. Un ejemplo de esta situación lo muestra 


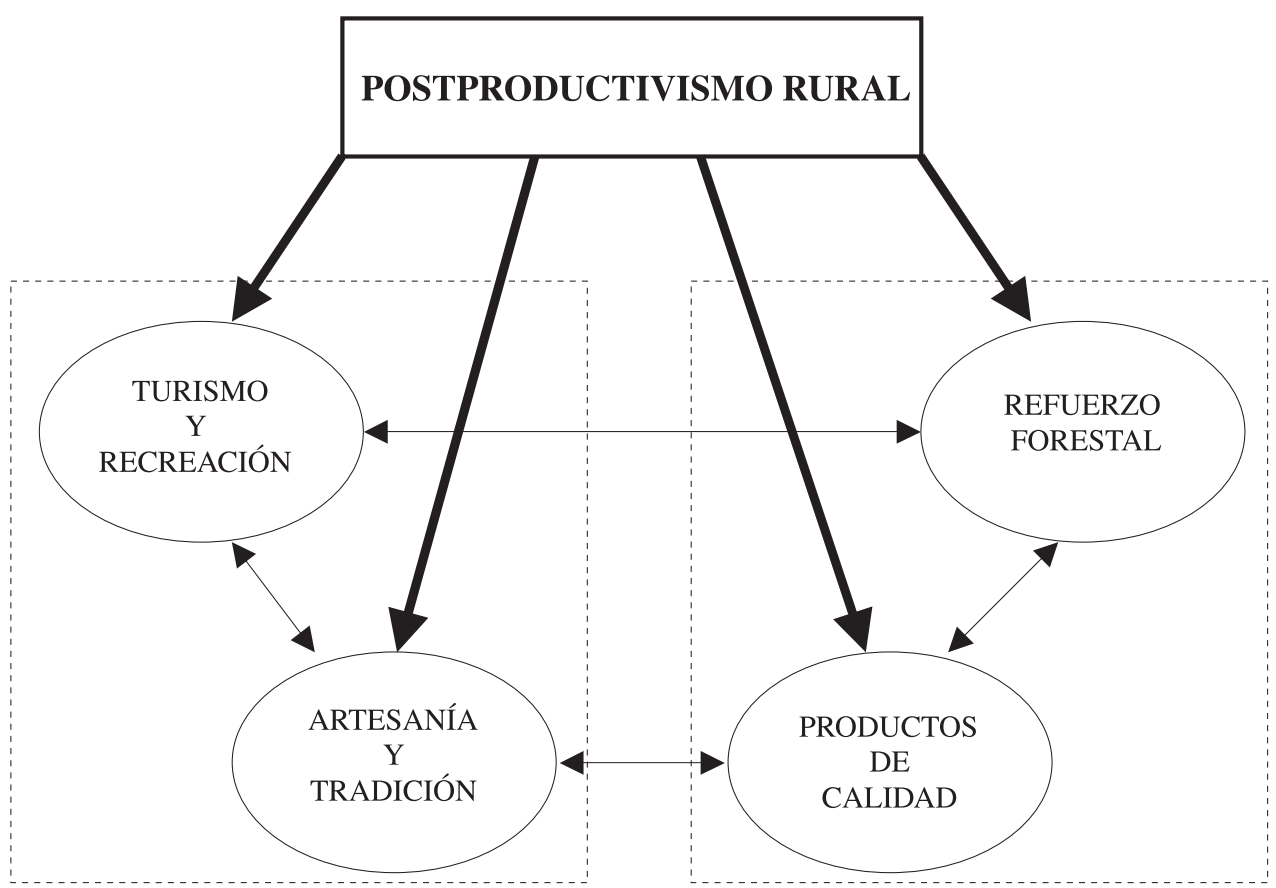

FIGURA 1. Los cuatro pilares del cambio postproductivista.

Benediktsson (2000) cuando explica que el turismo, que aparece por lo general como uno de los ejes en los que se debe apoyar la dimensión postproductivista del medio rural reforzando sus identidades, puede ser visto también como algo que perjudica la cultura local, ya que las tradiciones son reinventadas o incluso inventadas de nuevo para el disfrute del turista. Crang (ob. Cit. Benediktsson, 2000) hace al respecto un malicioso juego de palabras para definir esta situación con el término «fakelore». Íntimamente relacionado con esta crítica aparece el planteamiento esgrimido por Obón et al. (2000) quienes argumentan que para el caso del campo español en muchas ocasiones los Grupos de Desarrollo Local han considerado erróneamente al turismo como la única solución que le aporta una verdadera viabilidad.

Esta situación ha llevado a que se hable cada vez más de que el consumo de bienes rurales requiere de un diseño cuidadoso de las formas de regulación social. En los países industrializados las grandes corporaciones agroalimentarias y las organizaciones que orientan y prestan ayuda a la implantación de infraestructuras turísticas comienzan a estar más sensibilizadas sobre dónde y en qué condiciones sociales y ambientales están produciendo, fruto de la presión social y mediática a la que se han visto sometidas en los últimos años. Una realidad bien diferente es la actitud de esas empresas en los territorios periféricos.

Por tanto, en lo que respecta a la identificación de los beneficiarios del medio rural existen importantes variaciones según el análisis se localice en un sector productivista o en un área postproductivista. En el primer caso son los grandes propietarios y productores, las grandes empresas dedicadas al abastecimiento de insumos no agrarios, las grandes distribuidoras y comercializadoras, los consumidores con una capacidad adquisitiva más limitada y los residentes tradicionales los principales favorecidos. Además por lo general estos 
suelen ser los conformantes de los grupos de presión que poseen el poder de facto en el mundo rural. En el contexto postproductivista los más agraciados suelen ser los nuevos residentes, los visitantes, los consumidores habituales de productos de calidad, las agencias de viaje y las ONGs. Muchos de estos agentes enunciados en el segundo caso provienen geográficamente o formativamente del mundo urbano.

Tabla 1

BENEFICIARIOS DE LOS ENFOQUES ACTUALES DEL MEDIO RURAL

\begin{tabular}{|l|l|}
\hline Productivismo & Postproductivismo \\
\hline Productores & Productores \\
\hline Consumidores & Consumidores \\
\hline Residentes tradicionales & Residentes tradicionales \\
\hline Grandes propietarios & Residentes neorurales \\
\hline Grandes corporaciones & Propietarios \\
\hline & Visitantes \\
\hline & Agencias de Viaje \\
\hline & ONGs y Ecologistas \\
\hline
\end{tabular}

Marsden (1992) indica a éste respecto que en las áreas donde habitan residentes rurales tradicionales las actitudes y valores son marcadamente productivistas, mientras que en las que hay nuevos residentes el modelo postproductivista se extiende más. Es en esta última categoría en la que se pueden circunscribir la mayoría de los denominados «hobby-agricultores» que están interesados según Holloway (2000) en la producción alimentaria a pequeña escala como parte de un conjunto de decisiones que definen su estilo de vida en un contexto de viviendas rurales, de alimentos de calidad y de trabajo ético con animales. De hecho, es necesario señalar que la hobby-agricultura necesita del tejido urbano para sobrevivir, ya que por su propia definición, la fuente de ingresos principal del hobby-agricultor se sitúa fuera del sector primario, existiendo el trabajo fundamental en muchas ocasiones en el medio urbano.

\subsection{Los valores del medio ambiente y de la ruralidad}

El sustento de la actividad agraria han sido tradicionalmente el trabajo y la tierra, a pesar de que desde principios del siglo XX el trabajo ha disminuido por la mecanización y por las nuevas técnicas de gestión empresarial, y de que la tierra en las explotaciones más tecnificadas ha perdido su importancia, esta tierra todavía puede conservar múltiples valores para las personas. Esos valores dependieron históricamente de la relativa escasez o no del recurso, cosa que conllevó la competencia por la posesión, el uso y el manejo de ella. En la actualidad en los países desarrollados el mercado es el que decide la distribución de la tierra dependiendo de sus propias necesidades, siguiendo en parte las teorías de Von Thünen. La tierra junto con la concepción de recurso natural son los dos principios imperantes en el mundo de los economistas a la hora de valorar la función productiva de la naturaleza. 
Según Bergstrom (2002) los mercados de la tierra reflejan su valor como un insumo en la producción de mercancías económicas en la propia naturaleza de estos bienes privados, por tanto es posible cuantificarla económicamente de un modo tradicional dentro del proceso productivo. Esta visión contribuyó históricamente a enfatizar el productivismo en las áreas rurales desde las políticas agrarias y rurales. Los resultados de ese productivismo pueden comprobarse fácilmente en los paisajes rurales: a) campos dedicados a producir cantidades enormes de alimentos y de productos vegetales; b) bosques industriales privados donde los árboles destinados a crecer lo hacen con una precisión casi matemática. Este autor también señala, que en América el productivismo surgió como una forma de organizar y utilizar la tierra y otros insumos para hacer frente a las necesidades que la vida va planteando, derivándose de esta situación, tanto en los EE.UU. como en Europa, una preocupación social e individual por los excedentes producidos desde la industria agroalimentaria.

Lo problemático aparece cuando para algunos economistas de la actualidad (Simón, 1995; Bergstrom, 2002) hay que valorar de otro modo la tierra, de un modo que escapa de los mercados por intangible e inconmensurable, en inglés la expresión que define estos valores es amenity values cuyo significado aproximado sería algo así como valores de atractivo o valores ambientales ${ }^{15}$. Este concepto hay que inserirlo directamente en las corrientes postproductivistas rurales y conlleva como una de las características fundamentales, la recepción de visitantes de lo urbano que buscan aire y agua más puros en unos campos que tradicionalmente se habían definido desde la óptica productivista y que ahora están tomando un valor ambiental por el mero hecho de ser espacios abiertos. Ese valor visual que tienen la mayoría de los espacios rurales es un valor intangible, puesto que es difícil cuantificar el alcance económico del hábitat para la vida salvaje, de las vistas paisajísticas y de su generación de sentimientos de nostalgia basados en experiencias vividas real e imaginariamente o de la importancia de las construcciones típicas y el sentido de la comunidad rural para la sociedad en su conjunto. «Valorar económicamente el medio ambiente significa poder contar con un indicador de su importancia en el bienestar de la sociedad, que permita compararlo con otros componentes del mismo» (Azqueta, 1994: 11).

Naredo (ob. cit. Simón, 1995) argumenta que si bien, es de suma importancia la valoración de la naturaleza y su identificación con lo económico, no por eso se habrán encontrado soluciones a los problemas que se originan en la gestión medioambiental, entendiendo por solución algo definitivo que garantice la continuidad de la especie humana. Esta supervivencia, para Georgescu-Roegen (ob. cit. Simón, 1995), no es sólo económica ni sólo biológica, es bioeconómica, complicando por tanto, el modo de entender las relaciones bidireccionales entre el hombre y la naturaleza.

Para Murdoch y Miele (1999) hay dos maneras de entender la naturaleza: la primera es contemplarla como el cimiento de la última bondad que es posible encontrar, mientras que la segunda es verla como un recurso para la explotación humana. Siguiendo esta línea argumental, señalan que por una parte, la naturaleza es el objeto del conocimiento científico, y desde otra perspectiva se convierte en el objeto de devoción turística. Lo cierto es que estos espacios, a parte de la valoración económica ambiental, que se revela siempre como complicada, deben servir como atracción de nuevos visitantes y residentes.

Según Rosenberger y Walsh (ob. cit. Bergstrom, 2002) los valores ambientales se pueden dividir en tres categorías: a) los valores de espacio abierto que proporcionan beneficios visuales, de ocio y terapéuticos; b) los valores medioambientales que inciden en la protec-

15 En esta línea de valoración ambiental son interesantes las aproximaciones desde la economía de Azqueta (1994), Gonzague Pillet (1997) y de Jiménez Herrero (1997). 
ción de las aguas, suelos, hábitats animales y vegetales y de la biodiversidad; c) los valores de herencia cultural incluyendo el provecho de los suelos agrarios como parte de una única herencia o historia cultural y natural de un área. Aquí, la noción de biodiversidad, aparece de un modo restringido en su vertiente más conocida, la física, mientras que el concepto debe abarcar, en palabras de Lockwood (1999: 367), una dimensión más amplia, éticamente antropocéntrica, desde un punto de vista más emotivo, estético y espiritual con el objeto de «poder ayudar a salvar la vida en el planeta».

Ya a mediados de los años setenta David Pearce (ob. cit. Azqueta, 1994) identificaba cuatro funciones del medio ambiente en las que ya se pueden observar una mezcla de filosofía productivista y postproductivista: 1) forma parte de la función de producción de gran cantidad de bienes económicos en cuanto que consumen agua y aire de una determinada calidad; 2) actúa como un receptor de residuos y desechos fruto de la actividad productiva y consuntiva de la sociedad; 3) proporciona bienes naturales, como por ejemplo paisajes, que son demandados por la sociedad; 4) constituye un sistema integrado que proporciona los medios para sostener toda clase de vida.

Los valores actuales del medio rural y en consecuencia de las tierras agrarias son de cuatro tipos según Gardner (ob. cit. Bergstrom, 2002): a) producción de alimentos; b) provisión de puestos de trabajo; c) mejora de la organización de los usos del suelo; d) valores medioambientales. En la actualidad y bajo el enfoque postproductivista un fenómeno que tiene especial relevancia es la recalificación de la tierra agraria, pasando ésta de los usos considerados tradicionalmente como agrarios a usos no agrarios.

A estas aproximaciones teoréticas, en las que la validación económica se revela harto complicada, hay que añadirles otras perspectivas (Lockwood, 1999; Bergstrom, 2002) que enlazan la filosofía con el hecho religioso y espiritual. Los filósofos dedicados a analizar la cuestión ambiental diferencian entre dos tipos de valores:

a) instrumentales: dependen del uso del suelo activo o pasivo que sirve para generar o apoyar servicios que pueden ser útiles para las personas, animales, plantas o para los sistemas ecológicos en conjunto. Estos valores se dividen a su vez en económicos y no económicos;

b) intrínsecos ${ }^{16}$ : los que son independientes del uso pasivo o activo por parte de otras entidades, pudiendo representar este tipo de valores la tierra en esencia y los animales y plantas en sí mismos. Eso les ha llevado a hablar de tres visiones diferentes a la hora de analizar los valores intrínsecos: la primera es la denominada biocéntrica en la que todos los seres vivos tienen valor; la segunda es la ecocéntrica en la que poseen valor todos los elementos del paisaje sean o no seres vivos; y por último la llamada visión teocéntrica en la que aparecen los valores de los elementos vivos y no vivos como elementos de un dios independientemente de su uso activo o pasivo por parte de algo o alguien en la Tierra, de hecho se trata de una visión con carácter cosmogónico que ofrece variantes que pueden ir desde el monoteísmo occidental cristiano, el judaico o el islámico a formas como el budismo, el hinduismo o las religiones animistas en que todas las cosas tienen un valor de forma inherente que deriva de los sentimientos.

16 Otra visión del mismo asunto es la de Wounter de Groot (1992), los valores intrínsecos se dividen en: a) humanos, donde destacarían la salud y seguridad humana, el bienestar humano económico y el bienestar humano cultural y espiritual; b) del medio ambiente, donde hay que tener en cuenta el bienestar de la naturaleza; c) de las interrelaciones sociedad - medioambiente, donde el objetivo es la sostenibilidad y armonía entre el hombre y la naturaleza. (ob. cit. Jiménez, 1997). 
En el otro lado de la balanza surge, por tanto, la ética antropocéntrica, según la cual lo que le proporciona valor a las cosas, entre ellas la tierra y el medio ambiente, es la relación que las vincula con el ser humano, es el hombre el que le asigna una valoración a todo lo que le rodea, y a la que se hacía alusión en las páginas precedentes. Este substrato cultural de características antropocéntricas en el que el hombre aparece como dominador y señor de la naturaleza arranca con claridad en el siglo XVII con la figura de René Descartes. Bajo este prisma es desde el que se deben contemplar la mayoría de los análisis económicos, aunque algunos de los más prestigiosos economistas neoclásicos deben ser incluidos en sus planteamientos teóricos, dentro de lo que sería una filosofía protonaturista ${ }^{17}$. Estos valores instrumentales económicos, incluyen valoraciones sobre el consumo material, sobre el uso recreacional, sobre la apreciación escénica y sobre la salud.

Sin dejar con rotundidad las teorías sobre la valoración de la tierra agraria y el espacio rural, se puede apuntar con claridad que estos soportes cumplen una serie de funciones que se refuerzan o debilitan dependiendo según el contexto sea productivista o postproductivista. Estas funciones son al menos nueve:

1) el medio rural establece divisiones laborables entre los residentes tradicionales y los nuevos residentes, otorgándoles a cada uno de ellos una posición diferenciada;

2) las áreas rurales actúan como proveedoras de espacio;

3) el mundo rural provee hábitats para la flora y la fauna;

4) las tierras rurales proporcionan entornos físicos únicos;

5) los terrenos rurales son los que albergan el sistema natural de abastecimiento de agua siendo importantes tanto por la cantidad como por la calidad;

6) lo rural actúa como lugar de trabajo;

7) el medio rural provee a la sociedad de mercancías;

8) las áreas rurales proporcionan espacio para vivir;

9) el mundo rural funciona como un lugar para visitar.

\subsection{La perspectiva postproductivista en la agricultura}

En Europa occidental los dos modelos que definen la agricultura son las grandes explotaciones y la agricultura familiar tradicional. Esta identificación, simple y en cierto modo sesgada, puede servir por el momento para diferenciar los enfoques productivista y postproductivista. A este respecto Evans (2001) pone en duda que conceptualmente una ideología postproductivista haya reemplazado a otra productivista. Bajo esta perspectiva Peterson (2000) indica que hoy en día los sistemas productivista y postproductivista coexisten ${ }^{18}$. Benediktsson (2000) afirma acertadamente que las afirmaciones de que la época productivista ha muerto son exageradas. Mientras que Baraja (2001) refuerza este planteamiento al decir que el productivismo cumple una importante función en los campos europeos y no va a desaparecer de un plumazo porque haya aparecido un modo de producción alternativo. La coexistencia de los dos paradigmas indica que en la actualidad se ha de entender al sector agrario como multifuncional. Del mismo modo, esta cuestión lleva a Murdoch y Miele

17 A este respecto Azqueta (1994) señala como ejemplos los trabajos de Edgeworth y en lo que confiere a la economía clásica los de los utilitaristas Bentham y Stuart Mill.

18 De hecho, Ramos y Romero (1994) en un trabajo que analiza la política agraria de Andalucía indican que todavía a mediados de los ochenta el único enfoque que oficialmente existía en esta región era el productivista. La época en la que entra España en la CEE se puede definir como un período marcadamente productivista en la filosofía de la administración, los productores y los estudiosos. Aún hoy en día, la mayor parte de las tierras agrícolas de las regiones europeas están bajo ese planteamiento productivo. 
(1999) a establecer dos zonas de producción del sector alimentario contemporáneo: por un lado, las redes estandarizadas e industrializadas con carácter global, y por otro, los procesos de producción especializados y localizados territorialmente. Estos productos estandarizados y los especializados (o de calidad) son en cierto sentido mutuamente dependientes puesto que en el concepto de alimentos de calidad existe de manera inherente la definición de producto estándar.

Por lo que respecta a las grandes explotaciones, éstas sin duda, continuarán teniendo un destacado papel a escala europea. Los replanteamientos políticos de la Comisión Europea abogando por la sostenibilidad y la reducción de externalidades ${ }^{19}$ negativas, lejos de limitar el ámbito de actuación de los grandes propietarios y corporaciones lo que ha hecho es marginalizar todavía más las explotaciones que poseían de antemano unas limitaciones más grandes, a estas últimas parece que a priori, sólo les quede la solución de convertirse en reservas paisajístico-fotográficas. Aquí es donde aparece la tradicional falta de sintonía entre las políticas de defensa medioambiental y su aplicación práctica «Lo esencial en cualquier caso, es que quien genera una externalidad negativa no tiene que pagar por ello en un sistema de mercado, a pesar del perjuicio que causa; y que quien produce una externalidad positiva no se ve recompensado monetariamente. El resultado es, en definitiva, que el sistema de mercado produce demasiadas externalidades negativas, y menos externalidades positivas de las deseables» (Azqueta, 1994: 5) (Véase tabla 2). Así el reequilibrio territorial propuesto nominalmente por la Unión, en algunas regiones es sólo eso, nominal. Por otra parte, tampoco se puede obviar el importante papel que juegan económica y socialmente esas grandes empresas, puesto que desenvuelven una importante función, ocupando actualmente la mayoría de los segmentos de mercado de productos agroalimentarios que llegan hasta el consumidor.

En cuanto a las explotaciones familiares, la casuística es grande, se encuentran situaciones en las que su potencial es el de una gran explotación pero con un funcionamiento de estructura familiar. Se identifican estados en los que la viabilidad de estas explotaciones no es posible debido a su desfase espacio-temporal desde el punto de vis-

Tabla 2

EFECTOS PERNICIOSOS DE LA AGRICULTURA PRODUCTIVISTA

\begin{tabular}{|l|l|}
\hline Recurso & Efectos negativos \\
\hline Suelo & Erosión hídrica y eólica; degradación química, biológica y física \\
\hline Atmósfera & Cambio climático; polución \\
\hline Agua & Contaminación; sequías \\
\hline Vida salvaje & Mutaciones; disfuncionalidades fisiológicas; muerte \\
\hline Animales domésticos & Enfermedades físicas y mentales; extinción \\
\hline Cultivos & Pérdida de la diversidad genética; plagas \\
\hline Hombre & Disfuncionalidades fisiológicas, económicas y sociales; muerte \\
\hline
\end{tabular}

Fuente: Guzmán Casado et alt. (2000) Modificado.

19 Azqueta (1994) identifica externalidad como una economía externa o la actividad que un agente repercute sobre otro (directamente sobre él o sobre su capacidad de producción), sin que se pueda cobrar un precio por ello, en uno u otro sentido. 
ta económico y que llevan decenas de años desapareciendo de la escena económica y territorial; una posible solución, para algunas de las que quedan en activo, puede ser pasar a cumplir funciones medioambientales abandonando totalmente las productivas, aunque si como parece, se recortan las subvenciones y se aumenta el control de la actividad, difícilmente se podrán seguir manteniendo, ni siquiera con funciones paisajísticas. Finalmente hay explotaciones que se encuentran en una situación intermedia puesto que se hallan en una reorientación productiva o necesitan de ella para sobrevivir, reorientación que ha de pasar forzosamente por la aceptación de la multifuncionalidad, la diversificación de la actividad productiva, la diferenciación regional y la potenciación del concepto de calidad.

La diferenciación regional de los productos implica una reterritorialización, por tanto, una reimplantación de los procesos locales de producción ${ }^{20}$. Esta certificación territorial es denominada por Moran como la creación de la propiedad intelectual del espacio rural (ob. cit. Benediktsson, 2000), algo así como la aplicación de los derechos de autor en el mundo rural.

Dentro del término multifuncionalidad se incluye no sólo el carácter productivo en lo referente a alimentos, si no también la protección de la biodiversidad y de los paisajes rurales y la generación de empleos. Esta perspectiva alternativa del concepto proveniente de Europa, es totalmente diferente de la que otros sectores importantes en la negociación agraria internacional promulgan. Así, desde el denominado Grupo de Cairns se piensa que no es más que una cortina de humo para camuflar la continuación de las políticas agrícolas proteccionistas y que debe ser eliminada en el debate de la Organización Mundial para el Comercio (Potter y Burney, 2002). A pesar de estos desencuentros, y de que la PAC tiene una doble dirección (por un lado prima la intensificación, los pagos compensatorios no están pensados desde la perspectiva medioambiental, y por el otro anuncia a bombo y platillo que intenta realizar un campo multifuncional), el organigrama europeo parece seguir apostando en los foros internacionales por la multifuncionalidad como aspecto clave para su agricultura en el futuro.

En cuanto a la diversificación de las actividades productivas, a este fenómeno se le suele conocer con el nombre de pluriactividad, y es sin duda otro de los objetivos fundamentales de la transición postproductivista. En este caso, pueden producirse nuevas formas de localización del trabajo relacionadas con la participación del trabajo independiente de la explotación, pero que permiten al sujeto no tener que abandonar el medio rural. Ejemplos de estos empleos pueden ser los relacionados con la agroindustria, siempre y cuando sea cercana, con la artesanía o con el sector servicios (comercio, turismo, etc.). Kinsella et al. (2000) comentan que la pluriactividad no es algo nuevo y que ha sido tradicionalmente una estrategia de adaptación económica de los propietarios rurales para lograr la supervivencia cuando el mercado adquiría unas condiciones realmente duras, y que fue contemplada como un indicador de agricultura ineficiente y pobre. A este respecto Guzmán et al. (2000) introducen desde una perspectiva absolutamente postproductivista que la pluriactividad se ha de basar en la complementariedad de las actividades, recuperando las prácticas sustentables que históricamente se han realizado en el campo; y Bonnamour (2001) enuncia que las claves para que la diversificación tenga éxito son la iniciativa, el profesionalismo y la innovación, siendo este concepto fundamental para el porvenir del espacio rural europeo.

20 Para Obón et al. $(2000 ; 273)$ «El ámbito local se configura como el generador real de la potenciación y dinamización económica y social del territorio». 


\section{Reflexiones finales}

El postproductivismo como concepto diferenciado surge a principios de la década de los noventa del siglo pasado y es creado por los investigadores sociales británicos en la misma línea que otros conceptos como postfordismo o potmodernismo, así pues, su historia es todavía breve, y su aplicación en España no arranca hasta la segunda mitad de esa década a partir del contacto directo con los británicos.

La línea productiva agraria que se define con el postproductivismo es aquella en la que priman los aspectos asociados con el medioambiente y con la calidad de las propias producciones, aunque el postproductivismo no debe ser contemplado sólo desde esa perspectiva estrictamente agraria; el postproductivismo trasciende esa dimensión para convertirse en algo propiamente rural y como tal simboliza la aplicación de la multifuncionalidad. En este contexto los valores tradicionalmente asignados al campo como lugar de producción de bienes primarios son acompañados por otras funciones no menos importantes que ha de cumplir como son la provisión de empleo, la organización de los usos del suelo, el mantenimiento de la biodiversidad, el atractivo turístico, la preservación de patrones culturales y de los recursos naturales.

\section{Bibliografía}

ADDA, J. (1997): A mundialização da economia. Questões de economia, Ed. Terramar. Lisboa, $184 \mathrm{pp}$.

ALEXANDRATOS, N. (1995): Agricultura mundial hacia el año 2010. FAO y Ed. Mundiprensa. Madrid, $493 \mathrm{pp}$.

AZQUETA, D. (1994): Valoración económica de la calidad ambiental. Ed. McGraw Hill. Madrid, 299 pp.

BARAJA, E. (2001): «Los límites de la intensificación de las producciones agrícolas» en GARCÍA PASCUAL, F. (Ed.): El mundo rural en la era de la globalización: incertidumbres y potencialidades, pp.93-108, Serie Estudios, $\mathrm{n}^{\circ}$ 146, Ministerio de Agricultura, Pesca y Alimentación. Madrid.

BENEDIKTSSON, K. (2000): «Recreating rural spaces: Iceland's agricultural regions in a post-productivist era» en MOST CCPP Workshop. Joensuu, Finlandia.

BERGSTROM, J. (2002): «Postproductivism and rural land values» en Land Use Conflicts and Problems. Northeast Regional Center for Rural Development. Orlando, USA.

BONNAMOUR, J. (2001): «El mundo rural ante el nuevo siglo: retos y desafios» en GARCÍA PASCUAL, F. (Ed.): El mundo rural en la era de la globalización: incertidumbres y potencialidades, pp. 23-41, Serie Estudios, $\mathrm{n}^{\circ}$ 146, Ministerio de Agricultura, Pesca y Alimentación. Madrid.

BOWLER, I. R. (1992): «The industrialization of agriculture» en BOWLER, I. (Ed.): The Geography of Agriculture in Developed Market Economies. Longman Scientific and Technical, New York, USA, pp. 6-31 .

BOWLER, I. R. (1996): «Agricultural land use and the post-productivist transition» «en LÓPEZ ONTIVEROS, A. y MOLINERO HERNANDO, F. (Ed.): La investigación hispano-británica reciente en geografía rural: del campo tradicional a la transición postproductivista, pp. 179-187. Asociación de Geógrafos Españoles (AGE). Murcia.

CAMELO, A. (2000): «Génesis y apoteosis del postmodernismo» en Deslinde, edición electrónica http://www.deslinde.org.co

CHATTERTON, P. (2002): «Be realistic: demand the imposible. Moving towards strong sustainable development in an old industrial region» en Regional Studies, Vol. 36.5, pp. 552-561. 
COBB, D. et a.l (1999): «Interpretations of sustainable agriculture in the UK» en Progress in Human Geography, Vol. 23, n 2, pp. 209-235.

EVANS, N. (2001): «Reflexiones en torno al modelo agropecuario productivista» en GARCÍA PASCUAL, F. (2001): El mundo rural en la era de la globalización: incertidumbres y potencialidades, pp.45-64, Serie Estudios, $\mathrm{n}^{\circ}$ 146, Ministerio de Agricultura, Pesca y Alimentación. Madrid.

EVANS, N. y YARWOOD, R. (1996): «Rare breeds, livestock and the post-productivist country-side» en LÓPEZ ONTIVEROS, A. y MOLINERO HERNANDO, F. (Ed.): La investigación hispano-británica reciente en geografía rural: del campo tradicional a la transición postproductivista, pp. 133-148. Asociación de Geógrafos Españoles (AGE). Murcia.

GÓMEZ MENDOZA, J. (2001): «Las «nuevas» funciones socioeconómicas y medioambientales de los espacios rurales» en GARCÍA PASCUAL, F. (Ed.): El mundo rural en la era de la globalización: incertidumbres y potencialidades, pp.111-148, Serie Estudios, ${ }^{\circ}$ 146, Ministerio de Agricultura, Pesca y Alimentación. Madrid.

GUZMÁN CASADO, G. et al. (2000): Introducción a la agroecología como desarrollo rural sostenible. Ed. Mundi-Prensa. Madrid, $535 \mathrm{pp}$.

HALWEIL, B. (2002): «Una agricultura en interés de todos» en Worldwatch Institute: La situación del mundo 2002. Icaria Editorial. Barcelona, pp. 103-140.

HERVIEU, B. (1997): Los campos del futuro. Serie Estudios, $\mathrm{n}^{\circ} 118$, Ministerio de Agricultura, Pesca y Alimentación. Madrid, $195 \mathrm{pp}$.

HOLLOWAY, L. y ILBERY, B. (1997): «UK vining pea growers and freezers responses to potential global warming», en Tidschirft voor Economische Geographie, Vol. 88, $\mathrm{n}^{\circ} 3$, pp. $226-236$.

HOLLOWAY, L. (2000): «Hobby-Farming in the UK: producing pleasure in the Post-Productivist Countryside» en II Simposio Anglo-Español de Geografía Rural. Valladolid, (Ed. en CD-ROM).

ILBERY, B. (1994): «O desafio do abandono das terras» en PINDER, D. (1994): Europa occidental, desafíos e mudanças, Celta Ed. Oeiras, Portugal, pp. 275-293.

JIMÉNEZ HERRERO, L. M. (1997): Desarrollo sostenible y economía ecológica. Ed. Síntesis. Madrid, $365 \mathrm{pp}$.

KINSELLA, J. et al. (2000): «Pluriactivity as a livelihood strategy in Irish farm households and its role in rural development», en Sociología Ruralis, Vol. 40, n 40, pp. 481-496.

LOCKWOOD, J. A. (1999): «Agriculture and biodiversity: finding our place in this world» en Agriculture and Human Values, $\mathrm{n}^{\circ} 16$, pp. 365-379.

MARSDEN, T. (1992): «Exploring a rural sociology for the fordist transition», en Sociología Ruralis, Vol. 32, n 2/3, pp. 209-230.

MURDOCH, J. y MIELE, M. (1999): ««Back to Nature»: changing worlds of production in the food sector» en Sociología Ruralis, Vol. 39, nº 4, pp. 465-483.

OBÓN, Y. et al. (2000): «Agricultura y sostenibilidad en el marco de la agenda 2000», en COLOM, A. y BERTORZ, F. (2000): III Jornadas Internacionales de Desarrollo Rural Integrado, 1999, pp. 263-279. Centre Tecnològic Forestal de Catalunya. Solsona.

OLMOS, S. (2002): Climate Change research and rural studies: implications of the «Postproductivist Transition» and related trends for adaptation to climate change in agriculture», Department of Geography and Environmental Studies, Carleton University.

PETERSON, A. (2000): «Alternatives, traditions, and diversity in agriculture» en Agriculture and Human Values, $\mathrm{n}^{\circ} 17$, pp. 95-106.

PILLET, G. (1997): Economia ecológica, Introdução à economia do ambiente e recursos naturais. Instituto Piaget. Lisboa, 300 pp. 
POTTER, C. y BURNEY, J. (2002): «Agricultural multifunctionality in the WTO, legitimate non-trade concern or disguised protectionism?», en Journal of Rural Studies, Vol. $18, \mathrm{n}^{\circ} 1, \mathrm{pp} .35-47$.

RAMOS REAL, E. y ROMERO RODRÍGUEZ, J. J. (1994): «Del productivismo al ruralismo: una reflexión sobre la política agraria en Andalucía», en Revista de Estudios Agro-sociales, $\mathrm{n}^{\circ}$ 169, pp. 175-212. Ministerio de Agricultura y Pesca. Madrid.

REVELLI, M. (1996): «8 Tesis sobre el postfordismo» en Contrapoder, $\mathrm{n}^{\circ}$ 5, edición electrónica http://www.altediciones.com/t11.htm

RODRÍGUEZ, M. A. (1997): «Historia y postmodernismo» en Proyecto filosofía en español, edición electrónica http://www.filosofia.org

RUBIO TERRADO, P. (1999): «El postproductivismo en los espacios rurales» en GALDÓS URRUTIA, R. y RUIZ URRESTARAZU, E. (1999): Postproductivismo y medio ambiente. Informes Técnicos $\mathrm{n}^{\circ}$ 82. Publicaciones del Gobierno Vasco. Departamento de Agricultura y Pesca. Vitoria-Gasteiz, pp. $15-78$.

SAFÓN, V. (1996): «¿Del fordismo al postfordismo? El advenimiento de los nuevos modelos de organización industrial», en I Congreso de Ciencia Regional de Andalucía: Andalucía en el umbral del siglo XXI, pp. 310-318. Edición electrónica.

SANZ HERNÁNDEZ, A. (2001): «Desarrollo rural. ¿Ocio o necesidad?, (en línea) 5Campus.com. Sociología http://www.5campus.com/leccion/desarural (15/07/2002)

SIMÓN, X. (1995): «Economía ecológica, agroecología y desarrollo rural sostenible», en Agricultura y Sociedad, n ${ }^{\circ} 77$, pp. 199-236.

STOREY, D. (2000): «Continuity and change in rural Ireland», en II Simposio Anglo-Español de Geografía Rural. Valladolid, (Ed. en CD-ROM).

TRAUGER, A. (2001): «Women farmers in Minnesota and the Post-Productivist Transition» en The Great Lakes Geographer, Vol 8, n 2, pp. 53-66.

VELTZ, P. (1999): Mundialización, ciudades y territorios. Ed. Ariel. Barcelona, 254 pp. 